\title{
LARGE VALUES OF L-FUNCTIONS ON 1-LINE
}

\author{
ANUP B. DIXIT AND KAMALAKSHYA MAHATAB
}

\begin{abstract}
In this paper, we study lower bounds of a general family of $L$-functions on the 1-line. More precisely, we show that for any $F(s)$ in this family, there exists arbitrary large $t$ such that $F(1+i t) \geq e^{\gamma_{F}}\left(\log _{2} t+\log _{3} t\right)^{m}+O(1)$, where $m$ is the order of the pole of $F(s)$ at $s=1$. This is a generalization of the same result of Aistleitner, Munsch and the second author for the Riemann zeta-function. As a consequence, we get lower bounds for large values of Dedekind zeta-functions and Rankin-Selberg $L$-functions of the type $L(s, f \times f)$ on the 1-line.
\end{abstract}

\section{Introduction}

The growth of the Riemann zeta-function $\zeta(s)$ in the critical strip $1 / 2<\mathfrak{R}(s)<1$ has been of interest to number theorists for a long time. In this context, the upper bound is predicted by the Lindelöf hypothesis, which claims that $|\zeta(\sigma+i t)| \ll|t|^{\epsilon}$ for any $\epsilon>0$ and $1 / 2<\sigma<1$. This is, in fact a consequence of the famous Riemann hypothesis. Although there is significant progress towards this problem, no unconditional proof is known (see [22] for more details).

A more intricate question is to investigate how large $|\zeta(\sigma+i t)|$ can be for a fixed $\sigma \in[1 / 2,1)$ and $t \in[T, T+H]$, some interval. In this direction, Balasubramanian and Ramachandra [7] showed that

$$
\max _{t \in[T, T+H]}\left|\zeta\left(\frac{1}{2}+i t\right)\right| \geq \exp \left(c \sqrt{\frac{\log H}{\log _{2} H}}\right),
$$

where $c$ is a positive constant, $H \ll \log _{2} T$ and $\log _{2} T$ denotes $\log \log T$. From now on, we will denote $\log \log \ldots \log T$ by $\log _{k} T$. This result was improved by Bondarenko and Seip [9] in a

$k$ times

larger interval and was later optimized by Bretche and Tenenbaum [10, who showed that

$$
\max _{t \in[0, T]}\left|\zeta\left(\frac{1}{2}+i t\right)\right| \geq \exp \left((\sqrt{2}+o(1)) \sqrt{\frac{\log T \log _{3} T}{\log _{2} T}}\right) .
$$

For $\sigma \in(1 / 2,1)$ and $c_{\sigma}=0.18(2 \sigma-1)^{1-\sigma}$, Aistleitner [1] proved that

$$
\max _{t \in[0, T]}\left|\zeta\left(\frac{1}{2}+i t\right)\right| \geq \exp \left(c_{\sigma} \sqrt{\frac{\log T}{\log _{2} T}}\right) .
$$

On the other hand, we expect much finer results for large values of $L$-functions on $\mathfrak{R}(s)=1$. In [12], Granville and Soundararajan used techniques of diophantine approximation to show

2010 Mathematics Subject Classification. 11M41.

Key words and phrases. Dedekind zeta function, values on 1-line.

ABD is supported by the Coleman postdoctoral fellowship of Queen's university.

KM is supported by Grant 227768 of the Research Council of Norway and Project 1309940 of Finnish Academy. 
that

$$
\max _{t \in[0, T]}|\zeta(1+i t)| \geq e^{\gamma}\left(\log _{2} T+\log _{3} T-\log _{4} T+O(1)\right)
$$

for arbitrarily large $T$. This is an improvement on the previous bounds given by Levinson [14]. Granville and Soundararajan [12] conjectured that

$$
\max _{t \in[T, 2 T]}|\zeta(1+i t)|=e^{\gamma}\left(\log _{2} T+\log _{3} T+C_{1}+o(1)\right),
$$

where $C_{1}$ is an explicitly computable constant.

In 2017, Aistleitner, Munsch and the second author [2] used the resonance method to prove that there is a constant $C$ such that

$$
\max _{t \in[\sqrt{T}, T]}|\zeta(1+i t)| \geq e^{\gamma}\left(\log _{2} T+\log _{3} T+C\right) .
$$

Note that this result essentially matches (11), however, the size of the interval here is much larger. Unfortunately, over shorter intervals $[T, T+H]$, very little seems to be known regarding large values of $\zeta(1+i t)$ (see [5], 6] for further details).

In this paper, we generalize (2) to a large class of $L$-functions, namely $\mathbb{G}$, which conjecturally contains the Selberg class $\mathbb{S}$. We establish (2) for elements in $\mathbb{G}$ with non-negative Dirichlet coefficients. The key difference between $\mathbb{G}$ and $\mathbb{S}$ is that elements in $\mathbb{G}$ satisfy a polynomial Euler-product which is a more restrictive condition than that in $\mathbb{S}$. However, the functional equation in $\mathbb{S}$ is replaced by a weaker "growth condition" in $\mathbb{G}$. This is a significant generalization because most Euler products, which have an analytic continuation exhibit a growth condition, but perhaps not a functional equation. As applications, we prove the analogue of (2) for Dedekind zeta-functions $\zeta_{K}(s)$ and Rankin-Selberg $L$-functions given by $L(s, f \times f)$. We also prove a generalized Merten's theorem for $\mathbb{G}$ as a precursor to the proof of our main theorem.

The resonance method with a similar resonator was used by Aistleitner, Munsch, Peyrot and the second author [3] to establish large values of Dirichlet $L$-functions $L(s, \chi)$ with a given conductor $q$ at $s=1$. Perhaps, a similar method can also be used to establish large values over more general orthogonal families of $L$-functions in $\mathbb{G}$.

1.1. The class $\mathbb{G}$. In 1989 , Selberg [20] introduced a class of $L$-functions $\mathbb{S}$, which is expected to encapsulate all naturally occurring $L$-functions arising from arithmetic and geometry.

Definition 1.1 (The Selberg class). The Selberg class $\mathbb{S}$ consists of meromorphic functions $F(s)$ satisfying the following properties.

(i) Dirichlet series - It can be expressed as a Dirichlet series

$$
F(s)=\sum_{n=1}^{\infty} \frac{a_{F}(n)}{n^{s}}
$$

which is absolutely convergent in the region $\mathfrak{R}(s)>1$. We also normalize the leading coefficient as $a_{F}(1)=1$.

(ii) Analytic continuation - There exists a non-negative integer $k$, such that $(s-1)^{k} F(s)$ is an entire function of finite order. 
(iii) Functional equation - There exist real numbers $Q>0$ and $\alpha_{i} \geq 0$, complex numbers $\beta_{i}$ and $w \in \mathbb{C}$, with $\mathfrak{R}\left(\beta_{i}\right) \geq 0$ and $|w|=1$, such that

$$
\Phi(s):=Q^{s} \prod_{i} \Gamma\left(\alpha_{i} s+\beta_{i}\right) F(s)
$$

satisfies the functional equation

$$
\Phi(s)=w \bar{\Phi}(1-\bar{s}) .
$$

(iv) Euler product - There is an Euler product of the form

$$
F(s)=\prod_{p \text { prime }} F_{p}(s),
$$

where

$$
\log F_{p}(s)=\sum_{k=1}^{\infty} \frac{b_{p^{k}}}{p^{k s}}
$$

with $b_{p^{k}}=O\left(p^{k \theta}\right)$ for some $\theta<1 / 2$.

(v) Ramanujan hypothesis - For any $\epsilon>0$,

$$
\left|a_{F}(n)\right|=O_{\epsilon}\left(n^{\epsilon}\right) .
$$

The Euler product implies that the coefficients $a_{F}(n)$ are multiplicative, i.e., $a_{F}(m n)=$ $a_{F}(m) a_{F}(n)$ when $(m, n)=1$. Moreover, each Euler factor also has a Dirichlet series representation

$$
F_{p}(s)=\sum_{k=0}^{\infty} \frac{a_{F}\left(p^{k}\right)}{p^{k s}},
$$

which is absolutely convergent on $\mathfrak{R}(s)>0$ and non-vanishing on $\mathfrak{R}(s)>\theta$, where $\theta$ is as in $(i v)$.

For the purpose of this paper, we need a stronger Euler-product to ensure that the Euler factors factorize completely and further require a zero free region near 1-line, similar to what we notice in the proof of prime number theorem. However, we can replace the functional equation with a weaker condition on the growth of $L$-functions on vertical lines. This leads to the definition of the class $\mathbb{G}$.

Definition 1.2 (The class $\mathbb{G})$. The class $\mathbb{G}$ consists of meromorphic functions $F(s)$ satisfying (i), (ii) as in the above definition and further satisfies

(a) Complete Euler product decomposition - The Euler product in (4) factorizes completely, i.e.,

$$
F(s):=\prod_{p} \prod_{j=1}^{k}\left(1-\frac{\alpha_{j}(p)}{p^{s}}\right)^{-1}
$$

with $\left|\alpha_{j}\right| \leq 1$ and $\mathfrak{R}(s)>1$.

(b) Zero-free region - There exists a positive constant $c_{F}$, depending on $F$, such that $F(s)$ has no zeros in the region

$$
\mathfrak{R}(s) \geq 1-\frac{c_{F}}{\log (|\mathfrak{I}(s)|+2)},
$$

except the possible Siegel-zero of $F(s)$. 
(c) Growth condition - For $s=\sigma+i t$, define

$$
\mu_{F}^{*}(\sigma):=\inf \left\{\lambda>0:|F(s)| \ll(|t|+2)^{\lambda}\right\} .
$$

Then,

$$
\frac{\mu_{F}^{*}(\sigma)}{1-2 \sigma}
$$

is bounded for $\sigma<0$.

One expects $\mathbb{S}$ to satisfy conditions $(a)$ and $(b)$. In fact, the Riemann zeta-function, the Dirichlet $L$-functions, the Dedekind zeta-functions and the Rankin-Selberg $L$-functions can be all shown to satisfy conditions $(a)$ and $(b)$. Furthermore, for elements in $\mathbb{S}$ the growth condition $(c)$ is a consequence of the functional equation (3). However, it is possible to have $L$-functions not obeying a functional equation to satisfy the growth condition. One can consider linear combination of elements in $\mathbb{S}$ to see this. A family of $L$-functions based on growth condition was introduced by V. K. Murty in [17] and the reader may refer to [11] for more details on this family. Also the Igusa zeta-function, and the zeta function of groups have Euler products but may not have functional equation, which is discussed in [19].

1.2. The Main Theorem. In this paper, we produce a lower bound for large values of $L$ functions in $\mathbb{G}$ on the 1-line. For a meromorphic function $F(s)$ having a pole of order $m$ at $s=1$, define

$$
c_{-m}(F)=\lim _{s \rightarrow 1}(s-1)^{m} F(s) .
$$

Theorem 1.3. Let $F \in \mathbb{G}$ have non-negative Dirichlet coefficients $a_{F}(n)$ and a pole of order $m$ at $s=1$. Then, there exists a constant $C_{F}>0$ depending on $F(s)$ such that

$$
\max _{t \in[\sqrt{T}, T]}|F(1+i t)| \geq e^{\gamma_{F}}\left(\left(\log _{2} T+\log _{3} T\right)^{m}-C_{F}\right),
$$

where $\gamma_{F}=m \gamma+\log c_{-m}(F)$ and $\gamma$ is the Euler-Mascheroni constant.

In the above theorem, since $a_{F}(n) \geq 0$, we clearly have $m \geq 1$. This is important because if $F$ has no pole at $s=1$, it is possible for $F(s)$ to grow very slowly on the 1 -line.

As an immediate corollary, we get the following result for Dedekind zeta-functions $\zeta_{K}(s)$. Let $K / \mathbb{Q}$ be a number field. The Dedekind zeta-function $\zeta_{K}(s)$ is defined on $\mathfrak{R}(s)>1$ as

$$
\zeta_{K}(s):=\sum_{\mathbf{0} \neq \mathfrak{a} \subseteq \mathcal{O}_{K}} \frac{1}{(\mathbb{N a})^{s}}=\prod_{\mathfrak{p}}\left(1-\frac{1}{(\mathbb{N p})^{s}}\right)^{-1},
$$

where $\mathfrak{a}$ runs over all non-zero integral ideals and $\mathfrak{p}$ runs over all non-zero prime ideals of $K$. The function $\zeta_{K}(s)$ has an analytic continuation to the complex plane except for a simple pole at $s=1$. Furthermore, $\zeta_{K}$ satisfies properties $(a),(b),(c)$ and therefore $\zeta_{K} \in \mathbb{G}$. Thus, by Theorem 1.3, we have

Corollary 1. For a number field $K$, there exists a constant $C_{K}>0$ depending on $K$ such that

$$
\max _{t \in[\sqrt{T}, T]}\left|\zeta_{K}(1+i t)\right| \geq e^{\gamma_{K}}\left(\log _{2} T+\log _{3} T-C_{K}\right),
$$

where $\gamma_{K}=\gamma+\log \rho_{K}$, with $\rho_{K}$ being the residue of $\zeta_{K}(s)$ at $s=1$. 
The $L$-function associated to the Rankin-Selberg convolution of any two holomorphic newforms $f$ and $g$, denoted by $L(s, f \times g)$, is in the Selberg class. Moreover, it can also be shown that $L(s, f \times g) \in \mathbb{G}$. Here $f$ and $g$ are normalized Hecke eigenforms of weight $k$. It is known that if $L(s, f \times g)$ has a pole at $s=1$, then $f=g$. Hence, from Theorem [1.3, we have the following.

Corollary 2. For a normalized Hecke eigenform $f$, there exists a constant $C_{f}>0$ such that

$$
\max _{t \in[\sqrt{T}, T]}|L(1+i t, f \times f)| \geq e^{\gamma_{f}}\left(\log _{2} T+\log _{3} T-C_{f}\right),
$$

where $\gamma_{f}=\gamma+\log \rho_{f}$, with $\rho_{f}$ being the residue of $L(s, f \times f)$ at $s=1$.

The result obtained in Theorem 1.3 is a refined version of the bound established by Aistleitner-Pańkowski [4, which states that if $F(s)$ is in the Selberg class and satisfies the prime number theorem, namely,

$$
\sum_{p \leq x}\left|a_{F}(p)\right|=\kappa \frac{x}{\log x}+\mathcal{O}\left(\frac{x}{\log ^{2} x}\right),
$$

then for large $T$,

$$
\max _{t \in[T, 2 T]}|F(1+i t)|=\Omega\left((\log \log T)^{\kappa}\right) .
$$

Furthermore, since we are assuming the zero-free region in $\mathbb{G}$, using [13, Theorem 1], we have $\kappa=m$. Hence, we get a slightly more refined result than (8), but on a larger interval $[\sqrt{T}, T]$.

The poles of any element $F$ in the Selberg class $\mathbb{S}$ are expected to arise from the Riemann zeta-function. More precisely, if $F(s)$ has a pole of order $m$ at $s=1$, then $F(s) / \zeta(s)^{m}$ is expected to be entire and in $\mathbb{S}$. Thus, it is not surprising to expect the lower bound in Theorem 1.3 to be of the order $(\log \log T)^{m}$.

It is possible to generalize Theorem 1.3 to the Beurling zeta-function [8] by constructing a suitable resonator over Beurling numbers instead of integers. However, this will carry us far afield from our current focus. Hence, we relegate it to future research.

\section{Mertens' theorem for the class $\mathbb{G}$}

In 1874, Mertens [15] proved the following estimate for truncated Euler-product of $\zeta(s)$, which is also known as Mertens' third theorem given by

$$
\prod_{p \leq x}\left(1-\frac{1}{p}\right)^{-1}=e^{\gamma} \log x+O(1)
$$

where $\gamma$ denotes the Euler-Mascheroni constant. The analogue of Mertens' theorem for number fields was proved by Rosen [18, who showed that

$$
\prod_{\mathbb{N} \mathfrak{P}<x}\left(1-\frac{1}{\mathbb{N P}}\right)^{-1}=\rho_{K} e^{\gamma} \log x+O(1),
$$

where $\rho_{K}$ denotes the residue of $\zeta_{K}(s)$ at $s=1$. The Mertens theorem for the extended Selberg class satisfying conditions $(a)$ and $(b)$ was proved by Yashiro [23] in 2013. Following similar approach, one can establish Mertens' theorem for $\mathbb{G}$, where we replace the functional equation by the growth condition. However, Yashiro's paper [23] seems to be available only on arXiv. Hence, we include the proof for the sake of completeness. 
Theorem 2.1. Let $F(s) \in \mathbb{G}$. Suppose that $F(s)$ has a pole of order $m$ at $s=1$ and $c_{-m}(F)$ be as in (7). Then, for a constant $0<C_{F} \leq 1$,

$$
\prod_{p \leq x} \prod_{j=1}^{k}\left(1-\frac{\alpha_{j}(p)}{p}\right)^{-1}=c_{-m}(F) e^{\gamma m}(\log x)^{m}\left(1+O\left(e^{-C_{F} \sqrt{\log x}}\right)\right) .
$$

Proof. We closely follow the method of Yashiro [23]. Denote by

$$
F(1 ; x):=\prod_{p \leq x} \prod_{j=1}^{k}\left(1-\frac{\alpha_{j}(p)}{p}\right)^{-1} .
$$

Let

$$
\log F(s)=\sum_{n=1}^{\infty} \frac{b_{F}(n)}{n^{s}} .
$$

By the complete Euler product (6), we have $b_{F}(n)=0$ if $n \neq p^{r}$ and $b_{F}(n) \ll n^{\theta}$ for some $\theta<1 / 2$. Since

$$
b_{F}\left(p^{r}\right)=\frac{1}{r} \sum_{j=1}^{k} \alpha_{j}(p)^{r}
$$

we have $\left|b_{F}\left(p^{r}\right)\right| \leq k$. Write

$$
\begin{aligned}
\log F(1 ; x) & =\sum_{p \leq x} \sum_{r=1}^{\infty} \frac{b_{F}\left(p^{r}\right)}{p^{r}} \\
& =\sum_{n \leq x} \frac{b_{F}(n)}{n}+\sum_{\sqrt{x}<p \leq x} \sum_{p^{r}>x} \frac{b_{F}\left(p^{r}\right)}{p^{r}}+\sum_{p \leq \sqrt{x}} \sum_{p^{r}>x} \frac{b_{F}\left(p^{r}\right)}{p^{r}} .
\end{aligned}
$$

It is easy to estimate the second and third term above as follows.

$$
\sum_{\sqrt{x}<p \leq x} \sum_{p^{r}>x} \frac{b_{F}\left(p^{r}\right)}{p^{r}} \ll \sum_{\sqrt{x}<p \leq x} \sum_{r=2}^{\infty} \frac{1}{p^{r}} \ll \sum_{\sqrt{x}<p \leq x} \frac{1}{p^{2}} \ll \frac{1}{\sqrt{x}} .
$$

Also,

$$
\sum_{p \leq \sqrt{x}} \sum_{p^{r}>x} \frac{b_{F}\left(p^{r}\right)}{p^{r}} \ll \sum_{p \leq \sqrt{x}} \frac{1}{x} \ll \frac{1}{\sqrt{x}}
$$

From (9), we get

$$
\log F(1 ; x)=\sum_{n \leq x} \frac{b_{F}(n)}{n}+O\left(\frac{1}{\sqrt{x}}\right)
$$

Setting $(1 / \log x)=u$ and $e^{\sqrt{\log x}}=T$ and using Perron's formula, we get

$$
\sum_{n \leq x} \frac{b_{F}(n)}{n}=\frac{1}{2 \pi i} \int_{u-i T}^{u+i T} \frac{x^{s}}{s} \log F(1+s) d s+O\left(e^{-c_{F} \sqrt{\log x}}\right) .
$$

Let $u^{\prime}=C_{F} / \log T=C_{F} / \sqrt{\log x}$. Choosing $x$ sufficiently large, we can ensure that there are no Siegel zeros for $F(1+s)$ in the region $\left[-u^{\prime}, u\right]$. Hence from the condition $(b), F(1+s)$ has no zeros in the region $-u^{\prime} \leq \mathfrak{R}(s) \leq u$ and $|\Im(s)| \leq T$ and has a pole of order $m$ at $s=0$.

Consider the contour $C$ joining $u-i T,-u^{\prime}-i T,-u^{\prime}+i T$ and $u+i T$. By the residue theorem, we have

$$
\operatorname{Res}_{s=0}\left(\frac{x^{s}}{s} \log F(1+s)\right)=\frac{1}{2 \pi i} \int_{C} \frac{x^{s}}{s} \log F(1+s) d s .
$$


We now estimate the above integral. Suppose $s=\sigma+i t$. By the growth condition $(c)$, we have

$$
|F(s)| \ll|t|^{\mu_{F}(\sigma)}
$$

where $\mu(\sigma) \ll(1-2 \sigma)$. Thus, for our choice of $u$ and $u^{\prime}$, we get for $\sigma \in\left[-u^{\prime}, u\right]$

$$
\log F(1+\sigma+i T) \ll(\log T)^{2} .
$$

Hence, we have

$$
\begin{aligned}
\left|\frac{1}{2 \pi i} \int_{u+i T}^{-u^{\prime}+i T} \frac{x^{s}}{s} \log F(1+s) d s\right| & \ll\left|\frac{(\log T)^{2}}{T} \int_{u}^{-u^{\prime}} x^{\sigma} d \sigma\right| \\
& \ll(\log x) e^{-\sqrt{\log x}} \\
& \ll e^{-c_{F}^{\prime} \sqrt{\log x}}
\end{aligned}
$$

for some $0<c_{F}^{\prime}<1$. Similarly, we also get

$$
\left|\frac{1}{2 \pi i} \int_{-u^{\prime}+i T}^{u+i T} \frac{x^{s}}{s} \log F(1+s) d s\right| \ll e^{-c_{F}^{\prime} \sqrt{\log x}} .
$$

We use the following result due to Landau (see [16, p. 170, Lemma 6.3]) to esimate the other terms in (10).

Lemma 2.2. Let $f(z)$ be an analytic function in the region containing the disc $|z| \leq 1$, supposing $|f(z)| \leq M$ for $|z| \leq 1$ and $f(0) \neq 0$. Fix $r$ and $R$ such that $0<r<R<1$. Then, for $|z| \leq r$ we have

$$
\frac{f^{\prime}}{f}(z)=\sum_{|\rho| \leq R} \frac{1}{z-\rho}+O\left(\log \frac{M}{|f(0)|}\right)
$$

where $\rho$ is a zero of $f(s)$.

Let $f(z)=(z+1 / 2+i t)^{m} F(1+z+(1 / 2+i t)), R=5 / 6$ and $r=2 / 3$ in the above Lemma 2.2. Using the zero-free region $(b)$, we get

$$
\left|\log s^{m} F(1+s)\right| \ll \begin{cases}\log (|t|+4), & |t| \geq 7 / 8 \text { and } \sigma \geq-u^{\prime} \\ 1 & |t| \leq 7 / 8 \text { and } \sigma \geq-u^{\prime}\end{cases}
$$

We now have the estimate

$$
\begin{aligned}
\left|\int_{-u^{\prime}}^{-u^{\prime}+i T} \frac{x^{s}}{s} \log F(1+s) d s\right| & \ll \int_{0}^{T} \frac{x^{-u^{\prime}}}{|s|}\left(\left|\log s^{m}\right|+\left|\log s^{m} F(1+s)\right|\right) d t \\
& \ll e^{-c_{F}^{\prime \prime} \sqrt{\log x}},
\end{aligned}
$$

for some $0<c_{F}^{\prime \prime}<1$. Similarly, we also have

$$
\left|\int_{-u^{\prime}-i T}^{-u^{\prime}} \frac{x^{s}}{s} \log F(1+s) d s\right| \ll e^{-c_{F}^{\prime \prime} \sqrt{\log x}} .
$$

Using the estimates (11), (12), (13) and (14) in the Equation (10) and choosing $C_{F}=$ $\min \left(c_{F}, c_{F}^{\prime}, c_{F}^{\prime \prime}\right)$, we get

$$
\frac{1}{2 \pi i} \int_{u-i T}^{u+i T} \frac{x^{s}}{s} \log F(1+s) d s=\operatorname{Res}_{s=0}\left(\frac{x^{s}}{s} \log F(1+s)\right)+O\left(e^{-C_{F} \sqrt{\log x}}\right)
$$

Let $\mathcal{C}$ denote the circle of radius $u^{\prime}$ centered at 0 . Then,

$$
\frac{1}{2 \pi i} \int_{\mathcal{C}} \frac{x^{s}}{s} \log F(1+s) d s=\operatorname{Res}_{s=0}\left(\frac{x^{s}}{s} \log F(1+s)\right) .
$$


Hence, it suffices to estimate the above integral. Since $F(s)$ has a pole of order $m$ at $s=1$,

$$
c_{-m}(F)=\lim _{s \rightarrow 1}(s-1)^{m} F(s) \neq 0 .
$$

Writing $F(s+1)=\left(s^{-m}\right)\left(s^{m} F(s+1)\right)$, we get

$$
\frac{1}{2 \pi i} \int_{\mathcal{C}} \frac{x^{s}}{s} \log F(1+s) d s=-\frac{m}{2 \pi i} \int_{\mathcal{C}} \frac{x^{s}}{s} \log s d s+\log c_{-m}(F) .
$$

The integral on the right hand side is

$$
\begin{aligned}
\int_{\mathcal{C}} \frac{x^{s}}{s} \log s d s & =\int_{-\pi}^{\pi} \frac{x^{u^{\prime} e^{i \theta}}}{u^{\prime} e^{i \theta}}\left(\log u^{\prime} e^{i \theta}\right)\left(i u^{\prime} e^{i \theta}\right) d \theta \\
& =i\left(\log u^{\prime}\right) \int_{-\pi}^{\pi} e^{u^{\prime} e^{i \theta} \log x} d \theta-\int_{-\pi}^{\pi} \theta e^{u^{\prime} e^{i \theta} \log x} d \theta .
\end{aligned}
$$

By the series expansion of exponential function, we have

$$
\begin{aligned}
\int_{-\pi}^{\pi} e^{u^{\prime} e^{i \theta} \log x} d \theta & =\int_{-\pi}^{\pi} d \theta+\sum_{r=1}^{\infty} \frac{\left(u^{\prime} \log x\right)^{r}}{r !} \int_{-\pi}^{\pi} e^{i r \theta} d \theta \\
& =2 \pi
\end{aligned}
$$

Similarly,

$$
\begin{aligned}
\int_{-\pi}^{\pi} \theta e^{u^{\prime} e^{i \theta} \log x} d \theta & =\int_{-\pi}^{\pi} \theta d \theta+\sum_{r=1}^{\infty} \frac{\left(u^{\prime} \log x\right)^{r}}{r !} \int_{-\pi}^{\pi} \theta e^{i r \theta} d \theta \\
& =\sum_{r=1}^{\infty}\left(\frac{\left(u^{\prime} \log x\right)^{r}}{r !}\right)\left(\frac{(-1)^{r} 2 \pi}{i r}\right) \\
& =\frac{2 \pi}{i} \sum_{r=1}^{\infty} \frac{(-1)^{r}}{r !} \int_{0}^{u^{\prime} \log x} w^{r-1} d w \\
& =\frac{2 \pi}{i} \int_{0}^{u^{\prime} \log x} \frac{e^{-w}-1}{w} d w .
\end{aligned}
$$

But the Euler-Mascheroni constant $\gamma$ satisfies the identity

$$
\gamma=\int_{0}^{1} \frac{1-e^{-w}}{w} d w-\int_{1}^{\infty} \frac{e^{-w}}{w} d w
$$

Thus, we have

$$
\begin{aligned}
\int_{0}^{u^{\prime} \log x} \frac{e^{-w}-1}{w} d w & =\gamma+\int_{1}^{u^{\prime} \log x} \frac{d w}{w}-\int_{u^{\prime} \log x}^{\infty} \frac{e^{-w}}{w} d w \\
& =\gamma+\log \log x+\log u^{\prime}+O\left(e^{-C_{F} \sqrt{\log x}}\right) .
\end{aligned}
$$

Combining the estimates above (16)-(18), we get

$$
\log F(1 ; x)=\log c_{-m}(F)+m \gamma+m \log \log x+O\left(e^{-C_{F} \sqrt{\log x}}\right) .
$$

Taking exponential on both sides and using the fact that $e^{y}=1+O(y)$ for $|y|<1$, we are done. 


\section{Proof of the main theorem}

For $F \in \mathbb{G}$, define

$$
F(s ; Y):=\prod_{p \leq Y} \prod_{j=1}^{k}\left(1-\frac{\alpha_{j}(p)}{p^{s}}\right)^{-1}
$$

We use the following approximation lemma.

Lemma 3.1. For large $T$,

$$
F(1+i t)=F(1+i t ; Y)\left(1+O\left(\frac{1}{(\log T)^{10}}\right)\right)
$$

for $Y=\exp \left((\log T)^{10}\right)$ and $T^{1 / 10} \leq|t| \leq T$.

Proof. From the Euler product of $F(s)$, we have for $\mathfrak{R}(s)>1$,

$$
\log F(s)=-\sum_{p} \sum_{j=1}^{k} \log \left(1-\frac{\alpha_{j}(p)}{p^{s}}\right)=\sum_{p} \sum_{j=1}^{k} \sum_{l} \frac{\alpha_{j}(p)^{l}}{l p^{l s}} .
$$

Let $t_{0}>0$ and let $\alpha>0$ be any sufficiently large constant. Define

$$
\sigma_{0}:=\frac{1}{\alpha \log T}, \sigma_{1}:=\frac{1}{(\log T)^{20}} \text { and } T_{0}:=\frac{T^{1 / 10}}{2} .
$$

Applying Perron's summation formula as in [21, Theorem II.2.2], we get

$$
\int_{\sigma_{1}-i T_{0}}^{\sigma_{1}+i T_{0}} \log F\left(1+i t_{0}+s\right) \frac{Y^{s}}{s} d s=-\sum_{p \leq Y} \sum_{j=1}^{k} \log \left(1-\frac{\alpha_{j}(p)}{p^{1+i t_{0}}}\right)+O\left(\frac{1}{(\log T)^{10}}\right) .
$$

Now, we shift the path of integration to the left. By the zero-free region of $F \in \mathbb{G}$, the only pole of the above integrand in $\mathfrak{R}(s) \geq \sigma_{0}$ and $\mathfrak{I}(s) \leq T_{0}$ is at $s=0$. Therefore, we have

$$
\log F\left(1+i t_{0}\right)=-\sum_{p \leq Y} \sum_{j=1}^{k} \log \left(1-\frac{\alpha_{j}(p)}{p^{1+i t_{0}}}\right)+O\left(\frac{1}{(\log T)^{10}}+\int_{\mathcal{C}} \log F\left(1+i t_{0}+s\right) \frac{Y^{s}}{s} d s\right),
$$

where $\mathcal{C}$ is the contour joining $\sigma_{0}-i T_{0}, \sigma_{1}-i T_{0}, \sigma_{1}+i T_{0}$ and $\sigma_{0}+i T_{0}$. Since, $|\log F(\sigma+i t)| \ll \log t$ on $\mathcal{C}$, we get

$$
\int_{\sigma_{1}-i T_{0}}^{-\sigma_{0}-i T_{0}} \log F\left(1+i t_{0}+s\right) \frac{Y^{s}}{s} d s \ll \frac{\log T}{T^{1 / 10}}, \quad \int_{-\sigma_{0}+i T_{0}}^{\sigma_{1}+i T_{0}} \log F\left(1+i t_{0}+s\right) \frac{Y^{s}}{s} d s \ll \frac{\log T}{T^{1 / 10}},
$$

and

$$
\int_{-\sigma_{0}-i T_{0}}^{-\sigma_{0}+i T_{0}} \log F\left(1+i t_{0}+s\right) \frac{Y^{s}}{s} d s \ll \frac{(\log T)^{2}}{\exp \left(\frac{1}{\alpha}(\log T)^{9}\right)}
$$

where all implied constants are absolute. Substituting the bounds from (20) and (21) in (19), for $T^{1 / 10} \leq t_{0} \leq T$, we obtain

$$
\log F\left(1+i t_{0}\right)=-\sum_{p \leq Y} \sum_{j=1}^{k} \log \left(1-\frac{\alpha_{j}(p)}{p^{1+i t_{0}}}\right)+O\left(\frac{1}{(\log T)^{10}}\right) .
$$

Similarly we may argue when $t_{0}$ is negative. 
By Lemma 3.1, it suffices to show Theorem 1.3 for $F(1+i t ; Y)$. We closely follow the argument in [2]. Set

and for primes $p \leq X$ set

$$
X=\frac{1}{6}(\log T)\left(\log _{2} T\right)
$$

$$
q_{p}=\left(1-\frac{p}{X}\right)
$$

Also set $q_{1}=1$ and $q_{p}=0$ for $p>X$. Extend the definition completely multiplicatively to define $q_{n}$ for all integers $n \geq 1$. Now define

$$
R(t)=\prod_{p \leq X}\left(1-q_{p} p^{i t}\right)^{-1} .
$$

Then we have

$$
\begin{aligned}
\log (|R(t)|) & \leq \sum_{p \leq X}(\log X-\log p) \\
& =\pi(X) \log X-\vartheta(X),
\end{aligned}
$$

where $\pi(X)$ is the prime counting function and $\vartheta(X)$ is the first Chebyshev function. By partial summation, we know that

$$
\pi(X) \log X-\vartheta(X)=\int_{2}^{X} \frac{\pi(t)}{t} d t=(1+o(1)) \frac{X}{\log X} .
$$

By our choice of $X$, we get

$$
|R(t)|^{2} \leq T^{1 / 3+o(1)} .
$$

From the Euler product, $R(t)$ has the following series representation

$$
R(t)=\sum_{n=1}^{\infty} q_{n} n^{i t}
$$

and hence we get

$$
|R(t)|^{2}=\left(\sum_{n=1}^{\infty} q_{n} n^{i t}\right)\left(\sum_{n=1}^{\infty} q_{n} n^{-i t}\right)=\sum_{m, n=1}^{\infty} q_{m} q_{n}\left(\frac{m}{n}\right)^{i t} .
$$

We have

$$
F(1+i t ; Y)=\prod_{p \leq Y} \prod_{j=1}^{k}\left(1-\frac{\alpha_{j}(p) p^{-i t}}{p}\right)^{-1}
$$

Since $\left|\alpha_{j}(p)\right| \leq 1$, we get

$$
|F(1+i t ; Y)| \ll(\log Y)^{k} \ll(\log T)^{10 k}
$$

Set $\Phi(t):=e^{-t^{2}}$ and recall that its Fourier transform is positive. Using (22), we have

$$
\left.\left|\int_{|t| \geq T} F(1+i t ; Y)\right| R(t)\right|^{2} \Phi\left(\frac{\log T}{T} t\right) d t \mid \ll 1
$$

and

$$
\left.\left|\int_{|t| \leq \sqrt{T}} F(1+i t ; Y)\right| R(t)\right|^{2} \Phi\left(\frac{\log T}{T} t\right) d t \mid \ll T^{5 / 6+o(1)} .
$$

Using the fact that $q_{1}=1$ and the positivity of the Fourier coefficients of $\Phi$, we also have the following lower bound 


$$
\int_{\sqrt{T}}^{T}|R(t)|^{2} \Phi\left(\frac{\log T}{T} t\right) d t \gg T^{1+o(1)} .
$$

By a similar argument, again using the positivity of the Fourier coefficients, we have

$$
\int_{-\infty}^{\infty} F(1+i t ; Y)|R(t)|^{2} \Phi\left(\frac{\log T}{T} t\right) d t \geq \int_{-\infty}^{\infty} F(1+i t ; X)|R(t)|^{2} \Phi\left(\frac{\log T}{T} t\right) d t .
$$

So, we restrict ourselves to primes $p \leq X$ in the truncated Euler-product. This is to ensure both $R(t)$ and $F(1+i t ; X)$ have the terms with same $q$ 's.

Write $F(1+i t ; X)$ as

$$
F(1+i t ; X):=\sum_{n=1}^{\infty} a_{k} k^{-i t}
$$

where $a_{k} \geq 0$. This is because the Dirichlet coefficients of $F(s)$ are non-negative. Now define

$$
\begin{aligned}
I_{1} & :=\int_{-\infty}^{\infty} F(1+i t ; X)|R(t)|^{2} \Phi\left(\frac{\log T}{T} t\right) d t \\
& =\sum_{k=1}^{\infty} a_{k} \sum_{m, n=1}^{\infty} \int_{-\infty}^{\infty} k^{-i t} q_{m} q_{n}\left(\frac{m}{n}\right)^{i t} \Phi\left(\frac{\log T}{T} t\right) d t .
\end{aligned}
$$

We also define

$$
I_{2}:=\int_{-\infty}^{\infty}|R(t)|^{2} \Phi\left(\frac{\log T}{T} t\right) d t
$$

Notice that since we are working with truncated Euler-products, everything is absolutely convergent. Now, using the fact that the Fourier coefficients of $\Phi$ are positive and that $q_{n}$ are completely multiplicative, we get the inner sum of $I_{1}$ as

$$
\begin{aligned}
\sum_{m, n=1}^{\infty} \int_{-\infty}^{\infty} k^{-i t} q_{m} q_{n}\left(\frac{m}{n}\right)^{i t} \Phi\left(\frac{\log T}{T} t\right) d t & \geq \sum_{n=1}^{\infty} \sum_{k \mid m} \int_{-\infty}^{\infty} k^{-i t} q_{m} q_{n}\left(\frac{m}{n}\right)^{i t} \Phi\left(\frac{\log T}{T} t\right) d t \\
& =q_{k} \sum_{n=1}^{\infty} \sum_{r=1}^{\infty} \int_{-\infty}^{\infty} q_{r} q_{n}\left(\frac{r}{n}\right)^{i t} \Phi\left(\frac{\log T}{T} t\right) d t
\end{aligned}
$$

Thus, we have

$$
\begin{aligned}
\frac{I_{1}}{I_{2}} & \geq \sum_{k=1}^{\infty} a_{k} q_{k}=\prod_{p \leq X} \prod_{j=1}^{k}\left(1-\frac{\alpha_{j}(p)}{p} q_{p}\right)^{-1} \\
& =\left(\prod_{p \leq X} \prod_{j=1}^{k}\left(1-\frac{\alpha_{j}(p)}{p}\right)^{-1}\right)\left(\prod_{p \leq X} \prod_{j=1}^{k}\left(\frac{p-\alpha_{j}(p)}{p-\alpha_{j}(p) q_{p}}\right)\right)
\end{aligned}
$$

Using the generalized Merten's Theorem 2.1, we have

$$
\begin{aligned}
\prod_{p \leq X} \prod_{j=1}^{k}\left(1-\frac{\alpha_{j}(p)}{p}\right)^{-1} & =e^{\gamma_{F}}(\log X)^{m}+O(1) \\
& =e^{\gamma_{F}}\left(\log _{2} T+\log _{3} T\right)^{m}+O(1)
\end{aligned}
$$


The second product in (23) can be bounded as follows.

$$
\begin{aligned}
-\log \left(\prod_{p \leq X} \prod_{j=1}^{k}\left(\frac{p-\alpha_{j}(p)}{p-\alpha_{j}(p) q_{p}}\right)\right) & =-\left(\sum_{p \leq X} \sum_{j=1}^{k} \log \left(\frac{p-\alpha_{j}(p)}{p-\alpha_{j}(p) q_{p}}\right)\right) \\
& \ll \sum_{p \leq X} \frac{1}{X} \\
& \ll \frac{1}{\log X} .
\end{aligned}
$$

From (23), (24) and (25), we get

$$
\frac{I_{1}}{I_{2}} \geq e^{\gamma_{F}}\left(\log _{2} T+\log _{3} T\right)^{m}+O(1)
$$

In other words, we have

$$
\frac{\left.\left|\int_{\sqrt{T}}^{T} F(1+i t ; X)\right| R(t)\right|^{2} \Phi\left(\frac{\log T}{T} t\right) d t \mid}{\int_{\sqrt{T}}^{T}|R(t)|^{2} \Phi\left(\frac{\log T}{T} t\right) d t} \geq e^{\gamma_{F}}\left(\log _{2} T+\log _{3} T\right)^{m}+O(1) .
$$

Hence, we conclude

$$
\max _{t \in[\sqrt{T}, T]}|F(1+i t)| \geq e^{\gamma_{F}}\left(\left(\log _{2} T+\log _{3} T\right)^{m}-C_{F}\right)
$$

\section{REFERENCES}

[1] C. Aistleitner. Lower bounds for the maximum of the Riemann zeta function along vertical lines. Math. Ann., 365, no. 1-2, 473-496, 2016.

[2] C. Aistleitner, K. Mahatab, M. Munsch. Extreme values of the Riemann zeta function on the 1-line. Int. Math. Res. Not. IMRN, no. 22, 6924-6932, 2019.

[3] C. Aistleitner, K. Mahatab, M. Munsch, A. Peyrot. On large values of $L(\sigma, \chi)$. Q. J. Math., 70, no. 3, 831-848, 2019.

[4] C. Aistleitner, E. Pańkowski. Large values of L-functions from the Selberg class. J. Math. Anal. Appl., 446, no. 1, 345-364, 2017.

[5] L. Arguin and D. Belius, P. Bourgade, M. Radziwiłl, K. Soundararajan. Maximum of the Riemann zeta function on a short interval of the critical line. Comm. Pure and Applied Math, 72, no. 3, 500-535, 2019.

[6] Louis-Pierre Arguin, Frédéric Ouimet, Maksym Radziwiłł. Moments of the Riemann zeta function on short intervals of the critical line. arXiv:1901.04061 [math.NT].

[7] R. Balasubramanian, K. Ramachandra. On the frequency of Titchmarsh's phenomenon for $\zeta(s)$-III. Proc. Indian Acad. Sci., 86, 341-351, 1977.

[8] A. Beurling. Analyse de la loi asymptotique de la distribution des nombres premiers généralisés. I. Acta Math., 68, no. 1, 255-291, 1937.

[9] A. Bondarenko, K. Seip. Large greatest common divisor sums and extreme values of the Riemann zeta function. Duke Math. J., 166, 1685-1701, 2017.

[10] R. de la Bretèche, G. Tenenbaum. Sommes de Gál et applications. Proc. London Math. Soc. (3), 119 , no. 1, 104-134, 2019.

[11] A. B. Dixit. The Lindelöf class of L-functions. PhD Thesis, University of Toronto, 2018.

[12] A. Granville, K. Soundarajan. Extreme vaues of $|\zeta(1+i t)|$. The Riemann zeta function and related themes: papers in honour of Professor K. Ramachandra, Ramanujan Mathematical society Lecture Notes Series, 2, 65-80, 2006.

[13] J. Kaczorowski, A. Perelli, On the prime number theorem for the Selberg class, Arch. Math., 80, 255-263, 2003.

[14] N. Levinson. $\Omega$-theorems for the Riemann zeta-function. Acta Arithmetica, 20, 317-330, 1972.

[15] F. Mertens. Ein Beitrag zur analytischen Zahlentheorie. J. Reine Angew. Math., 78, 46-62, 1874.

[16] H. Montgomery, R. C. Vaughan. Multiplicative number theory. I. Classical theory. Cambridge Studies in Advanced Mathematics, 97, Cambridge University Press, Cambridge, 2007 
[17] V. K. Murty. The Lindelöf Class of $L$-functions. eds. L. Weng and M. Kaneko, The conference of $L$ functions, World Scientific publication, 165-174, 2007.

[18] M. Rosen. A generalization of Mertens' theorem. J. Ramanujan Math. Soc., 14, 1999.

[19] M. du Sautoy. Natural boundaries for Euler products of Igusa zeta functions of elliptic curves. Int. J. Number Theory, 14, no. 8, 2317-2331, 2018.

[20] A. Selberg. Old and new conjectures and results about a class of Dirichlet series. Collected Papers, 2, Springer-Verlag, Berlin Heidelberg New York, 47-63, 1991.

[21] G. Tenenbaum. Introduction to analytic and probabilistic number theory (English summary). Translated from the second French edition (1995) by C. B. Thomas. Cambridge Studies in Advanced Mathematics 46, Cambridge University Press, 1995.

[22] E. C. Titchmarsh. The theory of Riemann-Zeta function. Second edition, revised by D.R. Heath-Brown, Clarendon Press-Oxford, 1986.

[23] Y. Yashiro. Mertens' theorem and prime number theorem for Selberg class. arXiv:1311.0754v4.

Department of Mathematics and Statistics, Queen's University, Jeffrey Hall, 48 University Ave, Kingston, Canada, ON, K7L 3N8

E-mail address: anup.dixit@queensu.ca

Kamalakshya Mahatab, Department of Mathematics and Statistics, University of Helsinki, P. O. Box 68, FIN 00014 Helsinki, Finland

E-mail address: accessing.infinity@gmail.com, kamalakshya.mahatab@helsinki.fi 